\title{
Pressure Flow of Non-Newtonian Fluids between Eccentric Double Cylinders with the Inner Cylinder Rotating
}

Part 2: Experiment

\author{
By Noriyasu Mori*, Mitsuhiro Yagami**, Takaaki Eguchi**, Kiyoji Nakamura** and Akira Horikawa**, \\ Members, TMSJ \\ * Nara Technical College, Nara \\ **Department of Mechanical Engineering, Osaka University, Osaka
}

Based on the Journal of the Textile Machinery Society of Japan, Transactions, Vol. 38, No. 3, 747-752 (1985-3)

\begin{abstract}
The authors experimentally observed the pressure flow of non-Newtonian fluids between eccentric double cylinders with the inner cylinder rotating. Experiments were carried out to measure the pressure gradient, the angle of the secondary flow region and the pressure distribution on the outer cylinder using aqueous solutions of hydroxy ethyl cellulose (HEC). Results are summarized as follows: 1) Axial flow rate was enhanced by the rotation of inner cylinder. Denote the axial flow rate $Q_{s}$ and $Q_{r}$ respectively with the inner cylinder stationary and rotating. Then the larger was the difference between $Q_{s}$ and $Q_{r}$ the larger was the effect of eccentricity.

2) The angle of the secondary flow region increased with decreasing number of inner cylinder rotation and increasing axial pressure gradient.

3) Distribution of pressure on outer cylinder was affected by the axial flow rate, radius ratio and eccentricity.

4) Experimental results agreed well with numerical predictions. Thus the reliability of the numerical method used in the previous paper was confirmed.
\end{abstract}

\section{Introduction}

Flow of Newtonian fluids between eccentric double cylinders with rotating inner or outer cylinder was studied experimentally by Yamada et al. ${ }^{[1]-[6]}$ and Nakabayashi et al. ${ }^{[7]-[9]}$ They measured the distribution of pressure acting on the cylinders and compared it with theoretical prediction. Furthermore they carried out experiments on Taylor instability, turbulent flows and herical flows between eccentric cylinders. As far as non-Newtonian flows are concerned Taylor vortices between concentric cylinders was studied experimentally using dilute polymer solutions. ${ }^{[10]-[12]}$ However, no experimental studies of creeping flows between eccentric rotating cylinders of viscoelastic fluid with shear-thinning viscosity is available.

In a previous paper, ${ }^{[3]}$ the present authors used the second-order fluid model with variable coefficients to numerically calculated the pressure flow of non-Newtonian fluids between eccentric double cylinders with a rotating inner cylinder using the finite difference method with the following results:

1) Flow rate is enhanced by the rotation of the inner cylinder.

2) Axial and transverse velocity distribution affect each other.

3) The distribution of pressure acting on the outer cylinder is affected by the axial flow.

4) Elasticity of the fluid which affects the velocity field very little does affect the pressure distribution on the outer cylinder.

In view of above previous results we carry out experiments using a solution of hydroxy ethyl cellulose (HEC) in order to examine the validity of the numerical predictions made in the previous paper. ${ }^{[13]}$

\section{Axial Pressure Gradient and Secondary Flow}

\subsection{Experimental apparatus}

Figure 1 shows the schematic diagram of experimental apparatus used in the present study. The fluid flows from the reservoir through the annular space of eccentric cylinders pushed by air pressure controlled by a reducing valve. In order to measure the axial pressure gradient two pressure holes are provided on the outer cylinder $135.0 \mathrm{~mm}$ apart making the holes distant enough from the entrance of eccentric cylinders. The pressure loss is measured by a pressure transducer (TOYODA DD101K) connected to the two pressure holes.

The inner and outer cylinders are sealed by oil seals and supported by bearings. Rotational speed of the inner cylinder is regulated by means of a variable speed motor. Radius of the inner cylinder is $20.0 \mathrm{~mm}$ or $24.0 \mathrm{~mm}$, and that of the outer cylinder is $25.0 \mathrm{~mm}$ to make the radius ratio $\kappa$ equal 0.8 and 0.96 . An eccentric mechanism was used to support the outer cylinder so that eccentricity can be varied merely by rotating the outer cylinder support.

For a visual observation of secondary flow the outer cylinder was made transparent by use of acrylic pipe. The width of secondary flow was measured by observing trace of aluminum powder mixed in fluid. The fluid uses was aqueous solutions of HEC (1.0\% and $1.5 \%)$. These solutions are suitable for the present experiment, because their rheological properties are unaffected by shear deformation. Viscosity of $1.5 \%$ HEC solution derived from the 


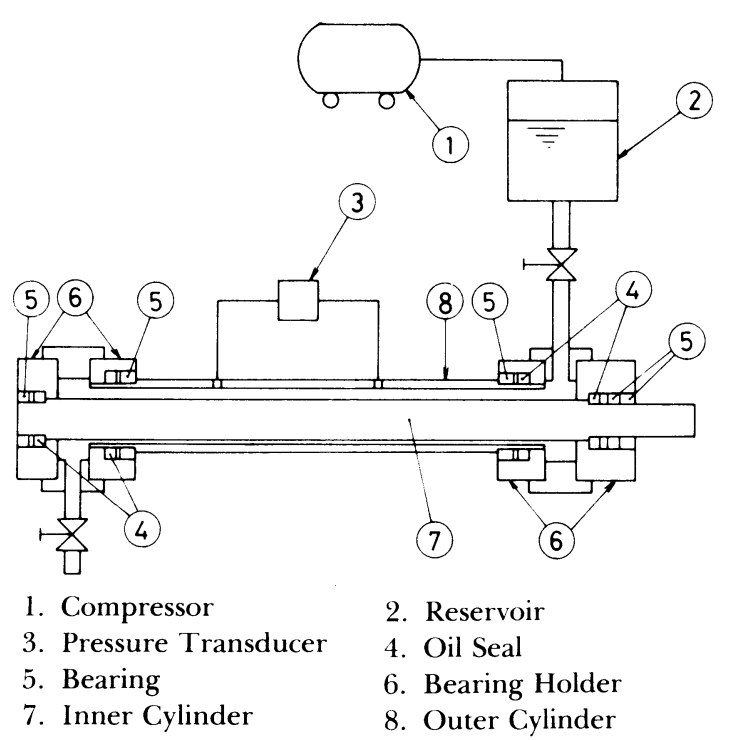

Fig. 1 Experimental apparatus for measuring the pressure loss and the width of secondary flow region

relation between flow rate and pressure gradient in concentric annular cylinder can be expressed by the fomula

$$
\eta=4.1\left(\frac{\operatorname{arcsinh}(1.3 \gamma)}{1.3 \gamma}\right)^{07} \mathrm{~Pa} \cdot \mathrm{s} \cdots \cdots(1)
$$

where $\gamma$ is the shear rate.

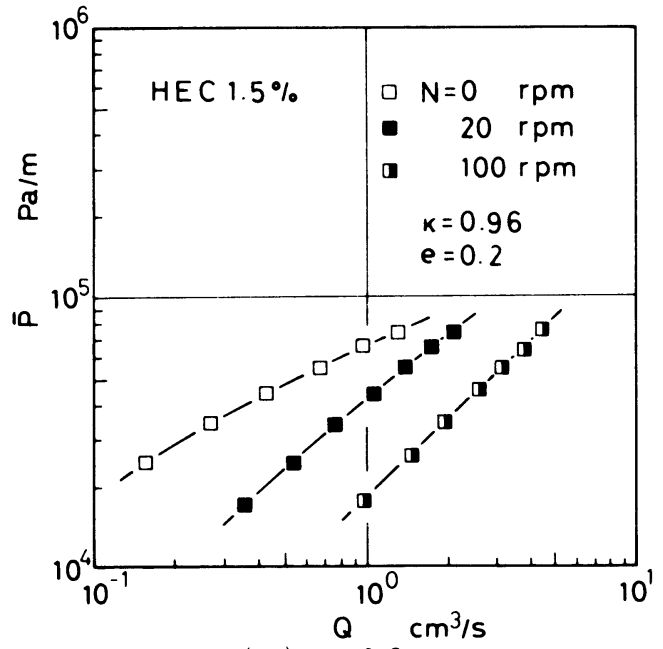

(a) $e=0.2$

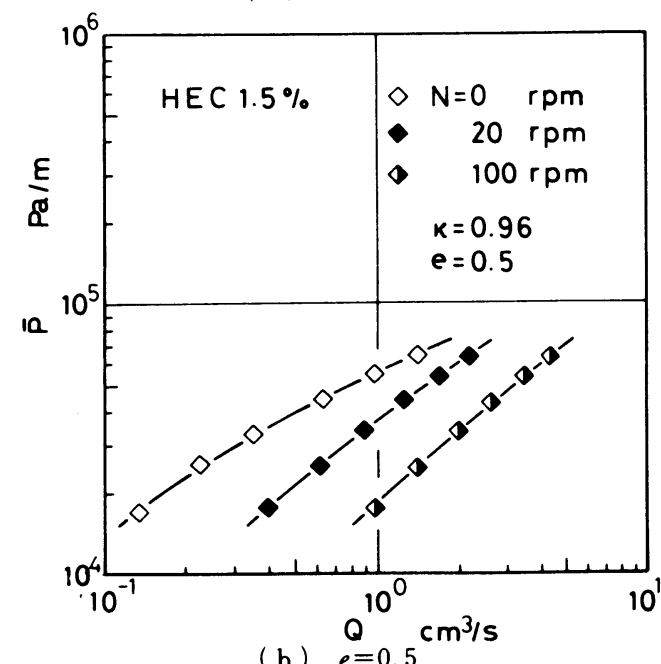

(b) $e^{Q}=0.5 \mathrm{~cm}$

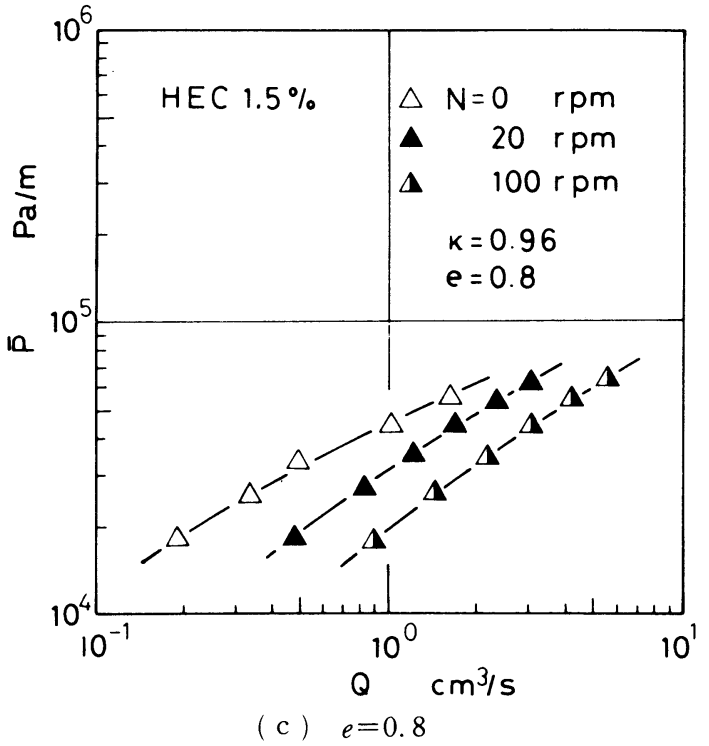

Fig. 2 Relation between flow rate and pressure gradient;

(a) $e=0.2$, (b) $e=0.5$, (c) $e=0.8$

\subsection{Measured pressure loss}

Figure 2 shows the relation between flow rate and pressure gradient for $1.5 \%$ HEC solution. Volume flow rate $Q$ always increases with increasing revolution $N$ of inner cylinder and the enhancement of flow rate $Q$ by revolution $N$ decreases as the pressure gradient becomes larger. As the three diagrams in Fig. 2 show the gradient of $\bar{P}$ versus $Q$ curves on log-log diagram is nearly independent of ec-

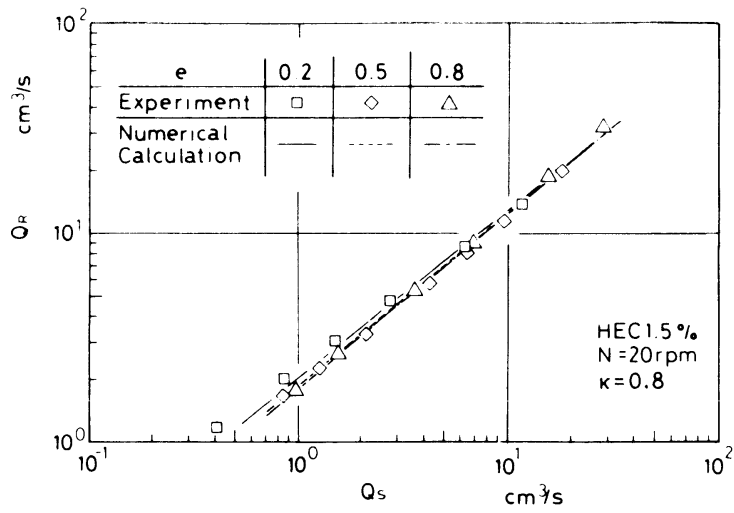

Fig. 3 Relation between $Q_{S}$ and $Q_{R}$ for $\kappa=0.8$; curves -----, - - - show numercial calculations in previous paper $^{[13]}$

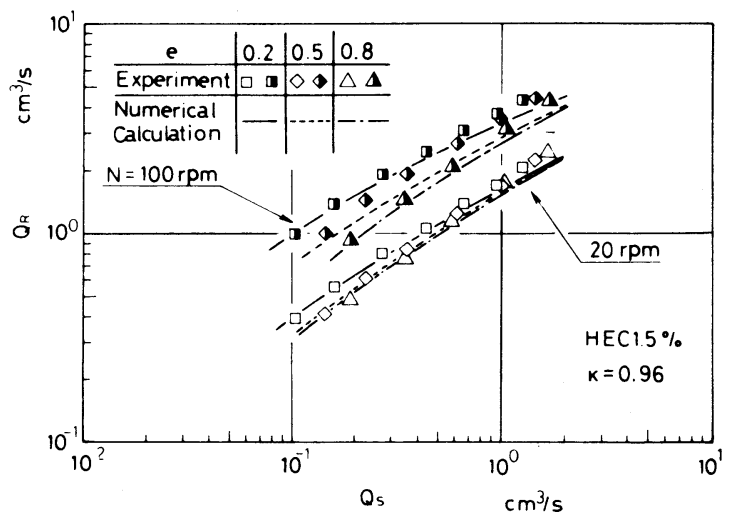

Fig. 4 Relation between $Q_{S}$ and $Q_{R}$ for $\kappa=0.96$; curves paper $^{[13]}-$ show numerical calculations in previous 
centricity $e$ when $N$ is equal to zero. As $N$ increases the $\bar{P}$ versus $Q$ curves becomes linear in shape and their gradient tends to decrease with increasing eccentricity $e$. To further clarify the effect of $e$ on $Q$ the relation between $Q$, and $Q$, under the same pressure gradient are shown in Figs. 3 and 4 where $Q_{s}$ and $Q_{r}$ are the flow rates under the stationary $(N=0)$ and the rotationaly $(N>0)$ condition of inner cylinder respectively. At the radius ratio of $\kappa=0.8$ the $Q_{\text {s versus }} Q_{r}$ diagram almost coincides with a straight line except at low flow rate. Note that the curves in Figs. 3 and 4 show numerical calculations neglecting elasticity terms using finite difference method and the viscosity formula eq. (1). Experimental values are in good quantitative agreement with numerical results. Consequently the reliability of the numerical method developed in the previous paper ${ }^{[13]}$ is experimentally verified. The $Q_{s}$ and $Q_{r}$ correlation for $\boldsymbol{\kappa}=0.96$ shown in Fig. 4 is practically unaffected by eccentricity $e$ at $N=20 \mathrm{rpm}$ as is the case in $\kappa=0.8$. At $N=100 \mathrm{rpm}$, however, $Q_{r}$ decreases with increasing $e$. For a fixed $Q_{s}$ the sensitivity of $Q_{r}$ to $N$ is considered to increase with increasing $N$. Therefore, it is reasonable to find $Q_{r}$ more sensitive to $e$ at high $N$.

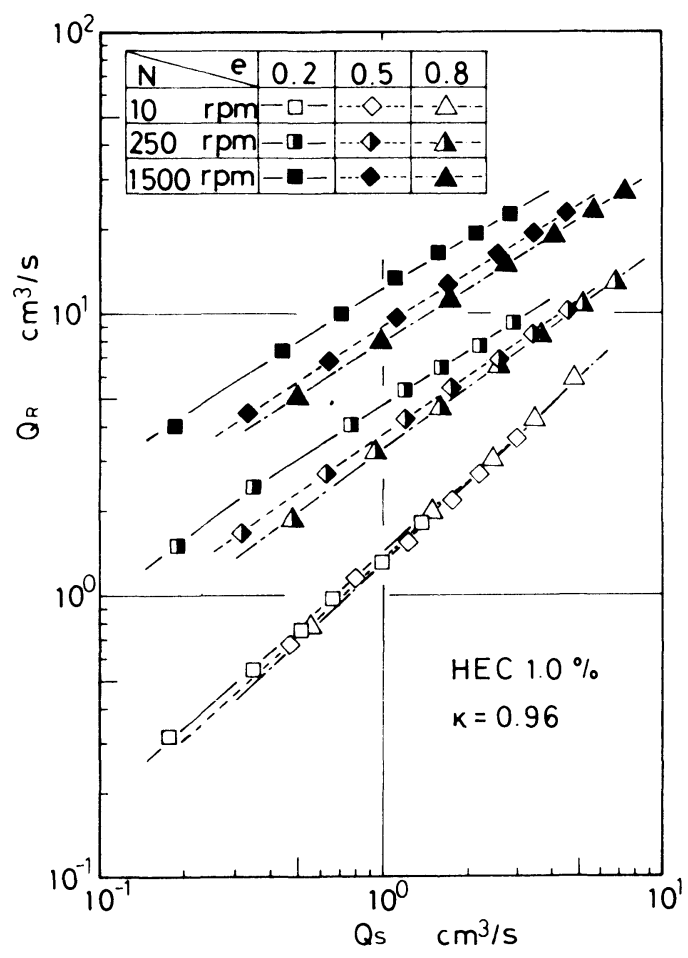

Fig. 5 Relation between $Q_{s}$ and $Q_{R}$ for $1.0 \%$ solution of HEC.

Figure 5 shows the relation between $Q_{s}$ and $Q_{r}$ for $1.0 \%$ HEC solution up to $N=1500 \mathrm{rpm}$ and Fig. 6 shows the relation between the number $N$ of rotations and the flow enhancement $I_{R}$ defined as

$$
I_{R}=\frac{Q_{R}-Q_{s}}{Q_{s}} \times 100 \quad(\%) \cdots \cdots \cdots \cdots \cdots(2)
$$

for $1.0 \%$ HEC solution.

The $I_{R}$ versus $N$ curves become linear at high $N$ values and the gradient of the curves is independent of eccentricity. For the same eccentricity, gradient of the curves becomes larger as $\mathrm{N}$ decreases or pressure gradient $\bar{P}$ increases.

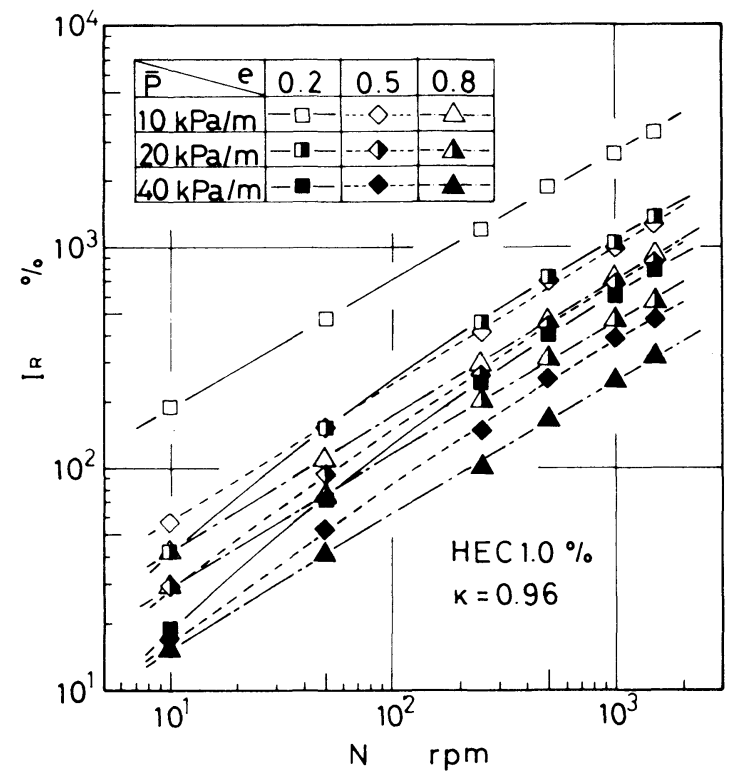

Fig. 6 Flow enhancement $I r$ as a function of the number of rotations $N$ for $1.0 \%$ solution of HEC.

\subsection{Width of secondary flow region}

In a previous paper ${ }^{[13]}$ the present authors predicted theoretically that the region of the secondary flow in nonNewtonian flow was narrower than that in Newtonian flows and the width varied with the number $N$ of rotation and axial How velocity. To experimentally verify the above prediction the authors measured the width of the region of secondary flow as the angle viewed from pipe center and compared with the numerical calculations.

Figure 7 shows schematically the paths of aluminum powder mixed in the fluid. Since the axial velocity is small relative to tangential velocity in the region of small clearance between the two cylinders aluminum powder moves
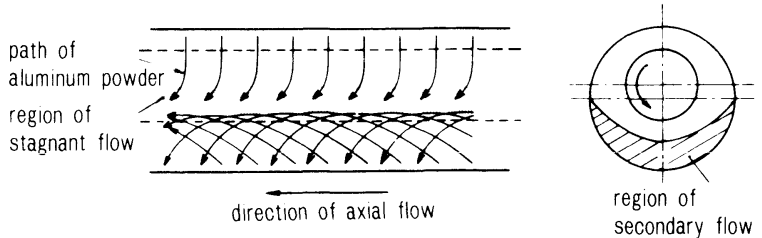

Fig. 7 A sketch of paths of aluminum powder

in the tangential direction. The movement of aluminum powder bends toward the axial direction as the clearance increases. In the region of secondary flow, the traces cross each other. Furthermore flow of aluminum powder stagnate near the end of secondary flow. Width of the secondary flow region was measured by taking the distance between the two ends of the region identified by the above stagnant flow and expressing the distance in aspect angle with respect to pipe center as shown in Fig. 8.

Table 1 shows the angle of secondary flow region under various conditions. Scatterings in the angle values result from the fact that the above stagnant flow spreads over a finite width. It is seen that the angle of the secondary flow region increases with decreasing number of rotation and increasing pressure gradient, but in general is considerably less than the theoretical prediction for Newtonian fluids. ${ }^{[14]}$ 
Table 1 A comparison of experimental data with numerical calculations from previous paper ${ }^{[14]}$

\begin{tabular}{|c|c|c|c|c|c|c|c|}
\hline$x$ & $e$ & $N(\mathrm{rpm})$ & $\bar{P}(\mathrm{kPa} / \mathrm{m})$ & $\begin{array}{l}\text { Experimental } \\
\text { Data }\left(^{\circ}\right)\end{array}$ & $\bar{P}(\mathrm{kPa} / \mathrm{m})$ & $\begin{array}{c}\text { Nume' } \\
\text { rical } \\
\text { Results } \\
\left({ }^{\circ}\right)\end{array}$ & $\begin{array}{l}\text { Theoretical. } \\
\text { Results for } \\
\text { N.F. }\left({ }^{\circ}\right)\end{array}$ \\
\hline \multirow{4}{*}{0.96} & \multirow{2}{*}{0.8} & 100 & $\begin{array}{l}7.99 \\
58.1\end{array}$ & $\begin{array}{l}230 \leftrightarrow 251 \\
239 \leftrightarrow 258\end{array}$ & $\begin{array}{r}0 \\
65\end{array}$ & $\begin{array}{l}204 \\
228 \\
\end{array}$ & \multirow{2}{*}{276.4} \\
\hline & & 20 & $\begin{array}{l}14.5 \\
62.4\end{array}$ & $\begin{array}{l}237 \leftrightarrow 256 \\
248 \leftrightarrow 265\end{array}$ & $\begin{array}{r}0 \\
65\end{array}$ & $\begin{array}{l}226 \\
238\end{array}$ & \\
\hline & \multirow{2}{*}{0.5} & 100 & $\begin{array}{l}5.44 \\
58.1\end{array}$ & $\begin{array}{l}0 \leftrightarrow 8 \\
0 \leftrightarrow 12\end{array}$ & $\begin{array}{r}0 \\
65\end{array}$ & $\begin{array}{r}0 \\
16\end{array}$ & \multirow{2}{*}{180.0} \\
\hline & & 20 & $\begin{array}{l}14.5 \\
76.2\end{array}$ & $\begin{array}{l}20 \leftrightarrow 42 \\
71 \leftrightarrow 89\end{array}$ & $\begin{array}{r}0 \\
65\end{array}$ & $\begin{array}{r}0 \\
66\end{array}$ & \\
\hline \multirow{2}{*}{0.8} & 0.8 & 20 & $\begin{array}{l}1.49 \\
3.99 \\
9.95\end{array}$ & $\begin{aligned} 255 & \leftrightarrow 273 \\
256 & \leftrightarrow 275 \\
256 & \leftrightarrow 276\end{aligned}$ & $\begin{array}{l}0 \\
1.5 \\
10\end{array}$ & $\begin{array}{l}242 \\
243 \\
250\end{array}$ & 281.4 \\
\hline & 0.5 & 20 & $\begin{array}{l}2.18 \\
5.23 \\
11.1\end{array}$ & $\begin{aligned} 86 & \leftrightarrow \\
97 & \leftrightarrow 117 \\
113 \leftrightarrow & \leftrightarrow 134\end{aligned}$ & $\begin{array}{l}0 \\
1.5 \\
10\end{array}$ & $\begin{array}{l}74 \\
86 \\
98\end{array}$ & 183.4 \\
\hline
\end{tabular}

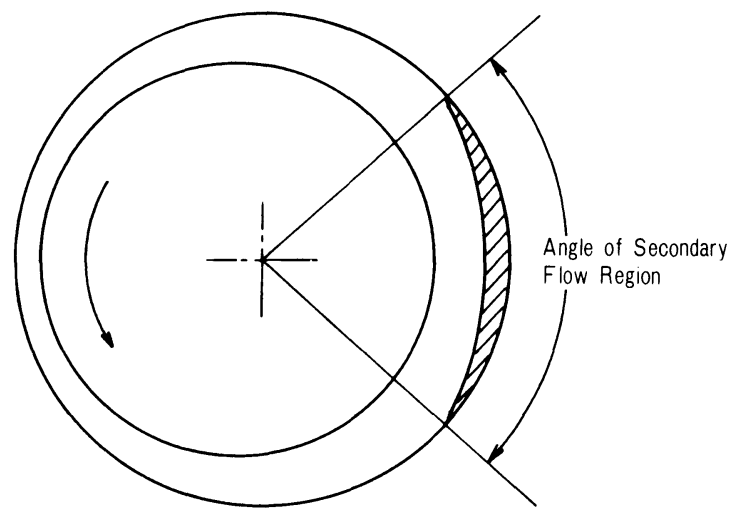

Fig. 8 Definition of the width of secondary flow region

\section{Distribution of Pressure on Outer Cylinder}

\subsection{Experimental apparatus}

In order to measure the peripheral pressure distribution on the outer cylinder, the third pressure hole was added half way between the two pressure holes for the measurement of pressure loss. A compact pressure transducer (TOYODA PD104K) was used on the third hole.

\subsection{Measured pressure distribution on outer cylinder}

The marked points in Fig. 9 show the pressure distribution $P_{0}-P_{r}$ over the outer cylinder periphery for $\boldsymbol{\kappa}=0.8$ and $N=20 \mathrm{rpm}$, where $P_{r}$ is the pressure on the outer cylinder at the maximum clearance and $\alpha_{0}$ is the angle between the radius of the outer cylinder at the maximum clearance and an arbitrary radius of the outer cylinder. Solid curves in Fig. 9 are the numerical prediction for $1.5 \%$ solution of HEC. ${ }^{[13]}$ In a previous paper ${ }^{[13]}$ it was shown that the pressure distribution $P_{0}-P_{r}$ on the outer cylinder exerted by non-Newtonian flows was affected by the axial flow, radius ratio $\boldsymbol{\kappa}$, eccentricity $e$ and other factors. As Fig. 9(a) for $e=0.2$ shows the pressure difference
$P_{0}-P_{r}$ varies more as the pressure gradient $\bar{P}$ becomes larger. For $e=0.5$, however, $P_{0}-P$, is little affected by axial flow. Furthermore at $e=0.8$ effect of pressure gradient $\bar{P}$ on the variation of $P_{0}-P_{r}$ is the opposite direction to that at $e=0.2$.

In the present experiments measurements for large flow-rates could not be made because of the small capacity of the tank. For this reaon the whole picture of the effect

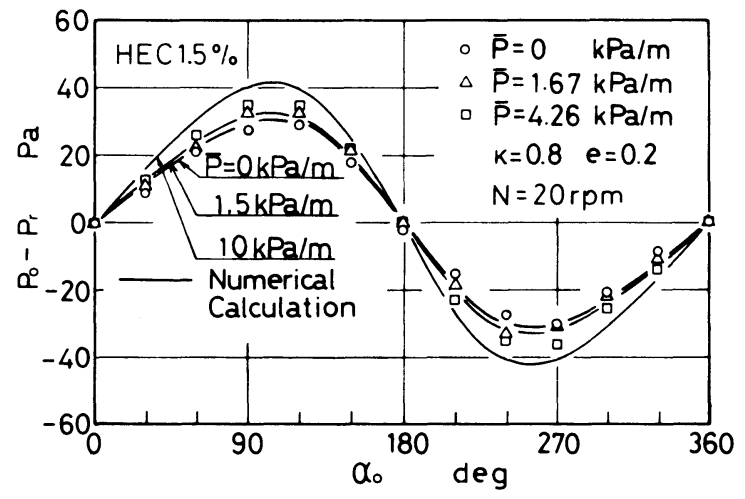

(a) $e=0.2$

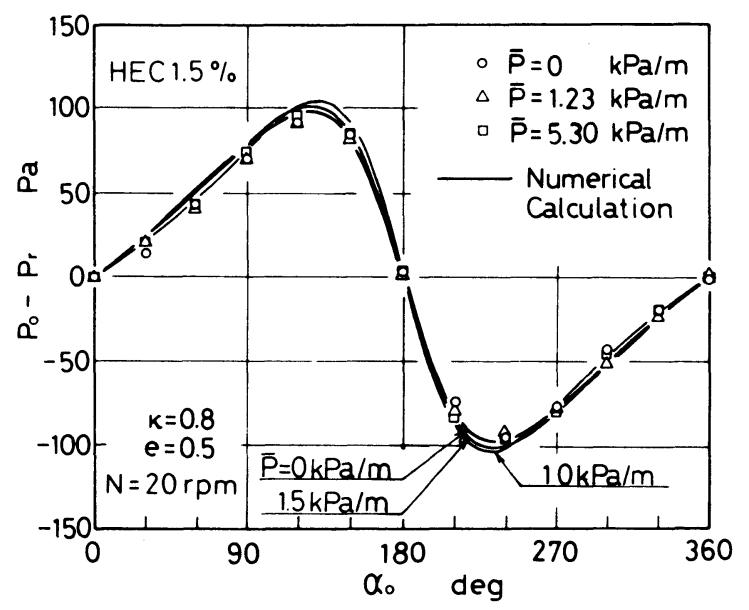

(b) $e=0.5$ 


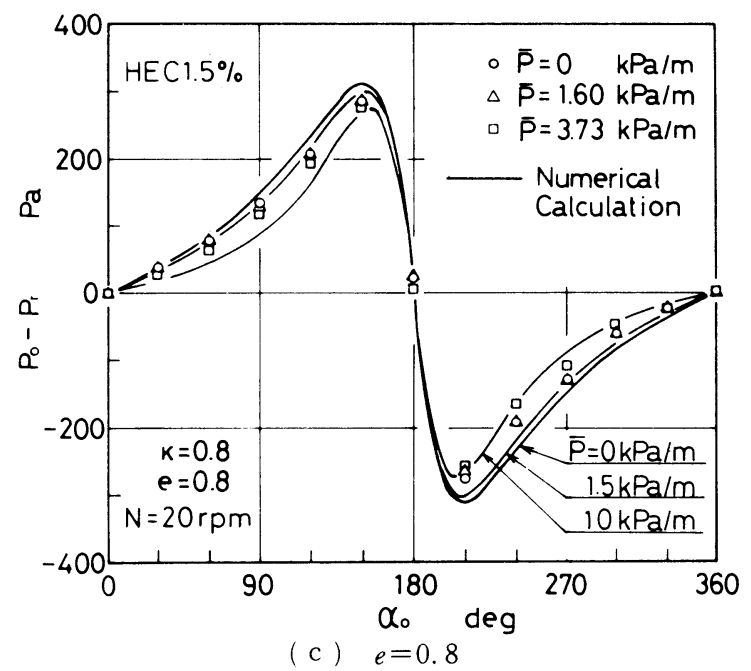

Fig. 9 Pressure difference distributions on outer cylinder for $\boldsymbol{\kappa}=0.8$; curves $\longrightarrow$ is numerical calculations in previous paper $^{[13]}$

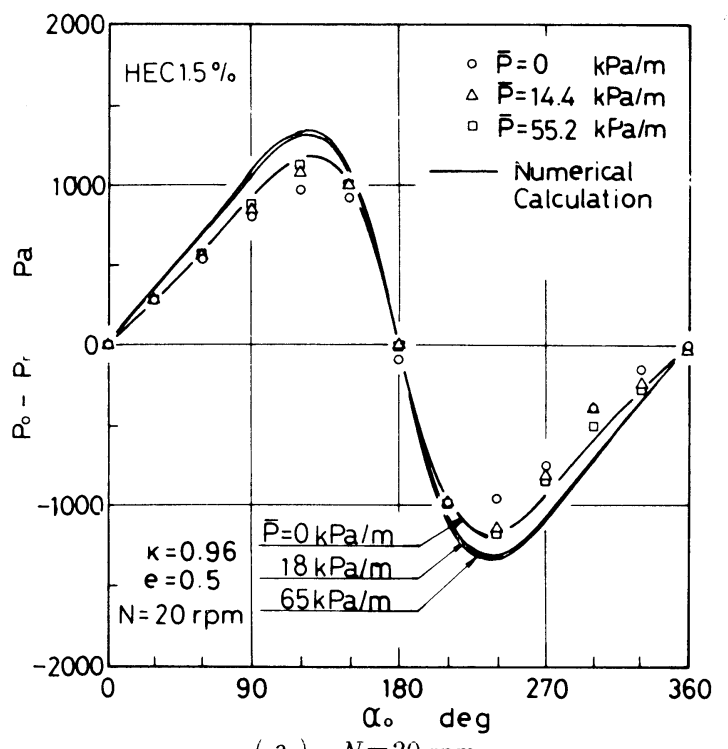

( a ) $N=20 \mathrm{rpm}$

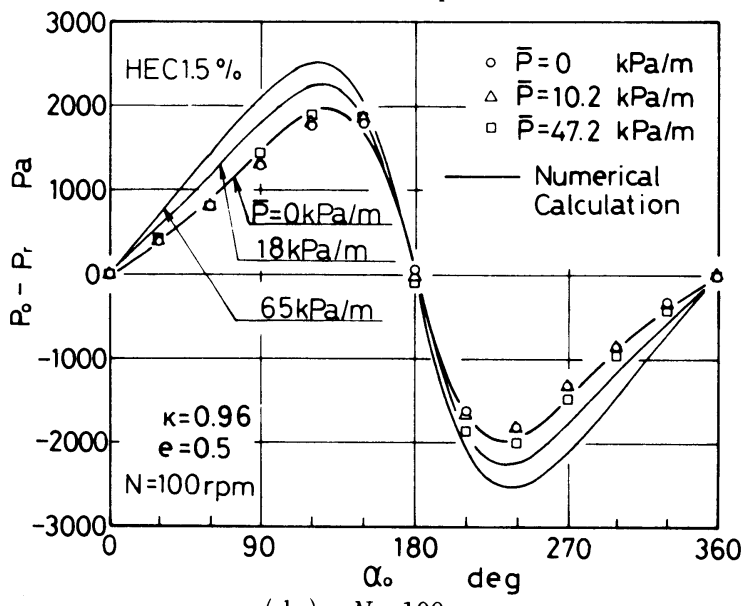

( b ) $\quad N=100 \mathrm{rpm}$

Fig. 10 Pressure difference distributions on outer cylinder for $\kappa=0.96$; curve - is numerical calculations in previous paper ${ }^{[13]}$ of pressure gradient on pressure difference distribution $P_{0}-P_{r}$ was not clarified. Within the range of present experiments, however, it appears that the measurements for $\boldsymbol{\kappa}=0.8$ agree well with numerical calculations. ${ }^{[13]}$

Figure 10 shows the measurements for the conditions $\kappa=0.96, e=0.5$ and $N=20 \mathrm{rpm}$ and $100 \mathrm{rpm}$. Measured variation of pressure difference $P_{0}-P_{r}$ is large when the pressure gradient is large to agree in trend with the numerical results. ${ }^{[13]}$

\section{Conclusions}

In a previous paper ${ }^{[13]}$ the distribution of axial velocity and pressure acting on the outer cylinder in the pressure flow of non-Newtonian fluids between eccentric double cylinders were calculated by means of finite difference method. In the present paper, the authors carried out experiments using aqueous solutions of HEC to verify the numerical calculations. ${ }^{[13]}$ It was found that experimental measurements agree well within numerical calculations that neglected elastic terms.

\section{References}

[1] Y. Yamada; Trans. Jpn. Soc. Mech. Eng., 27, 1267 (1961).

[2] Y. Yamada; Trans. Jpn. Soc. Mech. Eng., 28, 117 (1962).

[3] Y. Yamada, K. Nakabayashi; Trans. Jpn. Soc. Mech. Eng., 33, 1609 (1967).

[4] Y. Yamada et al.; Trans. Jpn. Soc. Mech. Eng., 35, 89 (1969).

[5] Y. Yamada, K. Nakabayashi; Trans. Jpn. Soc. Mech. Eng., 35, 1036 (1969).

[6] Y. Yamada, S. Watanabe; Trans. Jpn. Soc. Mech. Eng., 38, 1773 (1972).

[7] K. Nakabayashi et al.; Trans. Jpn. Soc Mech. Eng., 38, 2075 (1972).

[8] K. Nakabayashi et al.; Trans. Jpn. Soc. Mech.Eng., 10, 975 (1974).

[9] K. Nakabayshi, Y. Yamada; Trans. Jpn. Soc. Mech. Eng., 42, 3240 (1976).

[10] M. M. Denn, J. J. Roisman; AIChE Journal, 15, 454 (1969).

[11] Zu-Shung Sun, M. M. Denn; AIChE Journal, 18, 1010 (1972).

[12] W. M. Jones et al.; Rheol. Acta, 13, 798 (1974).

[13] N. Mori et al.; J. Text. Mach. Soc. Jpn., Trans., 38, T37 (1985).

[14] B. Y. Ballal, R. S. Rivlin; Trans. Soc. Rheol., 20, 65 (1976). 